\title{
Identification of Two Chemical Types of K21 Capsular Polysaccharide from Klebsiellae
}

\author{
By P. M. ALLEN, ${ }^{1}$ J. M. WILLIAMS,$^{3}$ C. A. HART ${ }^{2}$ AND \\ J. R. SAUNDERS ${ }^{1 *}$ \\ 1.2 Departments of Microbiology $y^{1}$ and Medical Microbiology ${ }^{2}$, Liverpool University, PO Box 147, \\ Liverpool L69 3BX, UK \\ ${ }^{3}$ Department of Physical Chemistry, Wellcome Research Laboratories, Langley Court, \\ Beckenham, Kent BR3 3BS, UK
}

(Received 13 August 1986; revised I December 1986)

\begin{abstract}
Strains of Klebsiella pneumoniae of serotype $\mathrm{K} 21$ are frequently involved in outbreaks of nosocomial infections. The type strain of Klebsiella pneumoniae K21 (which we have renamed $\mathrm{K} 21 \mathrm{a})$ produces capsular polysaccharide that contains mannose, galactose and glucuronic acid in the ratio $2: 2: 1$. In contrast, all eight of the randomly chosen isolates of Klebsiella pneumoniae that were initially typed as K 21 were shown by paper chromatography and NMR spectroscopy to produce a different capsular polysaccharide. We have designated this new polysaccharide $\mathrm{K} 21 \mathrm{~b}$. The K2lb capsular material appears to have a closely similar immunodominant side chain to K2la. However, K2lb polysaccharide has two molecules of rhamnose in the polysaccharide backbone replacing the two molecules of mannose found in the K21a capsule. Our results suggest that the K21b Klebsiella serotype may be more frequently distributed than the classical K21a type.
\end{abstract}

\section{INTRODUCTION}

Klebsiellae are Gram-negative bacilli that characteristically possess a thick polysaccharide capsule. The capsule is antigenic and can induce the production of specific anticapsular antibodies in mammals. At least 83 different capsular serotypes have been recognized (Nimmich, 1968). The capsules of klebsiellae are composed of heteropolymeric polysaccharide with a regular repeating oligosaccharide which often includes mono- or disaccharides as side chains. Sugar derivatives, characteristically glucuronic acid and pyruvylated or acetylated sugars are common constituents of the side chains. The chemical structure of the repeated oligosaccharide unit has been determined for many of the serotypes (Sutherland, 1977). Little diversity exists between the component monosaccharide units of the polysaccharides, variation being achieved by the differential arrangement of the sugar units within the oligosaccharide. However, the limited number of monosaccharides results in many capsules possessing regions of similarity with those from other serotypes. The presence of ketylated, acetylated or acid radicals in particular sugars is an important determinant of the antigenic specificity of a particular polysaccharide. The sugar unit contributing most to the specificity is termed the immunodominant sugar (Luderitz et al., 1966) and this sugar may be terminal or lie within the polysaccharide chain. In branched polysaccharides the immunodominant sugars usually reside within the side chains, which are thus antigenically more important than the backbone. Cross-reactivity between the different capsular antigens of klebsiellae can be correlated with the possession of similar side chains within the oligosaccharide unit. 
Klebsiellae are important nosocomial pathogens, primarily causing urinary tract and wound infections (Meers et al., 1981). The pathogenicity of klebsiellae appears to vary between different strains, and serotypes K2 and K21 are much more frequently involved in outbreaks of nosocomial infections than other serotypes (Casewell \& Talsania, 1979). The structure of the capsular polysaccharide of Klebsiella type K21 has been elucidated (Choy \& Dutton, 1973) and contains mannose, galactose and glucuronic acid in the ratio $2: 2: 1$. However, we have found a number of klebsiellae of serotype $\mathrm{K} 21$ that appear to possess a different capsular polysaccharide structure.

\section{METHODS}

Bacteria. The Klebsiella pneumoniae (K. aerogenes) K21 strain U53/1 (redesignated 1L918) was obtained from J. G. Barr, Royal Victoria Hospital, Belfast, Northern Ireland (Barr, 1981). The reference K. pneumoniae K21 (designated 2L222) and seven other randomly chosen Klebsiella K21 isolates (designated 2L225-2L231 respectively) were obtained from the Coventry Public Health Laboratory and the Central Public Health Laboratory (Colindale). The isolates originated in Britain, West Germany and Switzerland.

Extraction of polysaccharide. Bacteria were grown on minimal medium, comprising $0.4 \mathrm{~g} \mathrm{NH}_{4} \mathrm{Cl}, 7.5 \mathrm{~g}$ $\mathrm{Na}_{2} \mathrm{HPO}_{4}, 3.0 \mathrm{~g} \mathrm{KH}_{2} \mathrm{PO}_{4}, 0.5 \mathrm{~g} \mathrm{NaCl}, 0.2 \mathrm{~g} \mathrm{MgSO}_{4}, 0.01 \mathrm{~g} \mathrm{CaCl}_{2}$ and $10 \mathrm{~g}$ agar in 1 litre distilled water. The cells were then harvested and resuspended in distilled water. Extraction of polysaccharide was by the method of Wilkinson et al. (1955). The bacterial capsules were removed by boiling for $10 \mathrm{~min}$ and the suspension was centrifuged at $20000 \mathrm{~g}$ for $30 \mathrm{~min}$. The supernatant was retained and the capsular polysaccharide was precipitated with 2 vols acetone. The polysaccharide was resuspended to form a $0.5 \%(\mathrm{w} / \mathrm{v})$ solution in acetate buffer $[4 \%(\mathrm{w} / \mathrm{v})$ sodium acetate, $2 \%(\mathrm{w} / \mathrm{v})$ acetic acid, $\mathrm{pH} \mathrm{4.8]} \mathrm{and} \mathrm{was} \mathrm{then} \mathrm{deproteinized} \mathrm{by} \mathrm{shaking} 100 \mathrm{ml}$ volumes with $20 \mathrm{ml}$ chloroform and $4 \mathrm{ml}$ butan-1-ol. The polysaccharide was then reprecipitated with 2 vols acetone, dialysed extensively against tap water, precipitated and freeze dried. Polysaccharide was also extracted by the method of Gotschlich et al. (1969). All steps were performed at $0-4{ }^{\circ} \mathrm{C}$. The bulk of the nucleic acids and soluble protein were precipitated by adding $1 / 3 \mathrm{vol}$. absolute ethanol. The precipitate was sedimented by centrifugation at $20000 \mathrm{~g}$ for $20 \mathrm{~min}$ and the supernatant retained. Three volumes of ethanol were added and the precipitated polysaccharide was collected by centrifugation at $3000 \mathrm{~g}$ for $10 \mathrm{~min}$. The polysaccharide was deproteinized by shaking with chloroform and butan-1-ol as before, and dialysed against $0.1 \mathrm{M}-\mathrm{CaCl}_{2}$ for $24 \mathrm{~h}$. The polysaccharide solution was centrifuged at $100000 \mathrm{~g}$ and 3 vols ethanol were added. The precipitated polysaccharide was collected by centrifugation and dried in vacuo.

Hydrolysis. (a) The polysaccharide was hydrolysed in $2 \mathrm{M}-\mathrm{HCl}$ for $3 \mathrm{~h}$ at $100^{\circ} \mathrm{C}$. The hydrolysate was then rotary evaporated and resuspended in $20 \mu \mathrm{l}$ distilled water. Monosaccharide internal controls were similarly hydrolysed and included in chromatography experiments to determine if destruction of monosaccharides had occurred.

(b) Polysaccharide was partially hydrolysed at $\mathrm{pH} 2.3$ for $3 \mathrm{~d}$ at $95^{\circ} \mathrm{C}$ in an attempt to remove the side chain of the oligosaccharide repeating unit. The hydrolysate was rotary evaporated and dried in vacuo.

Chromatography. Hydrolysates were applied to Whatman 3MM chromatography paper and run in butan-1ol/acetic acid/water $(4: 1: 5$, by vol.) solvent. The papers were developed with alkaline silver nitrate to allow detection of sugars (Anonymous, 1974).

Gel diffusion technique for immunoprecipitation. Agarose $(1.1 \%$, w/v, in $0.05 \mathrm{M}$-barbitone buffer $\mathrm{pH} 8.6)$ was pipetted to form a thin layer on a glass slide. Wells were punched into the gel using a $2 \mathrm{~mm}$ diameter cork borer. A $15 \mu \mathrm{l}$ volume of K21 antiserum (obtained from Coventry Public Health Laboratory) was pipetted into the central well and $15 \mu \mathrm{l}$ capsular polysaccharide solution $(0.1 \%, \mathrm{w} / \mathrm{v}$, in distilled water) was pipetted into the surrounding wells. The gel was incubated in a humid chamber for $48 \mathrm{~h}$ at room temperature and then any unprecipitated proteins were eluted by soaking the gel in normal saline for a further $48 \mathrm{~h}$. The gel was dried and stained with Coomassie brilliant blue for $1 \mathrm{~h}$ and then destained for $10 \mathrm{~min}$. The gel was then examined for the presence of precipitin lines.

NMR spectroscopy. For NMR measurement the different samples of polysaccharide were each dissolved in $\mathrm{D}_{2} \mathrm{O}$, with heating when necessary. $\mathrm{K} 2 \mathrm{lb}$ polysaccharide that had been partially hydrolysed dissolved without warming.

' $\mathrm{H}$ NMR spectra were measured at $360 \mathrm{MHz}$ on a Bruker AM360 NMR spectrometer at both $30^{\circ} \mathrm{C}$ and $80^{\circ} \mathrm{C}$. Free induction decays (FIDs) were acquired into $32 \mathrm{~K}$ data points using $60^{\circ}$ pulses and a repetition rate of $3.3 \mathrm{~s}$. The accumulated FID was multiplied by an exponential function equivalent to a line broadening of $0.2 \mathrm{~Hz}$ before Fourier transformation. Lorentzian-Gaussian transformation of the FID was used to resolve spin couplings in some instances. Chemical shifts in the transformed spectra were referenced to acetate impurity at $\delta 1.95$ and in spectra of the partially hydrolysed $\mathrm{K} 2 \mathrm{lb}$ polysaccharide to internal acetone at $\delta 2 \cdot 225$.

The ${ }^{13} \mathrm{C}$ NMR proton decoupled spectrum of the partially hydrolysed $\mathrm{K} 21 \mathrm{~b}$ polysaccharide was measured at $90 \mathrm{MHz}$ at approximately $30^{\circ} \mathrm{C}$. Chemical shifts were externally referenced to dioxan in $\mathrm{D}_{2} \mathrm{O}$ at $67.4 \mathrm{p} . \mathrm{p} . \mathrm{m}$. 


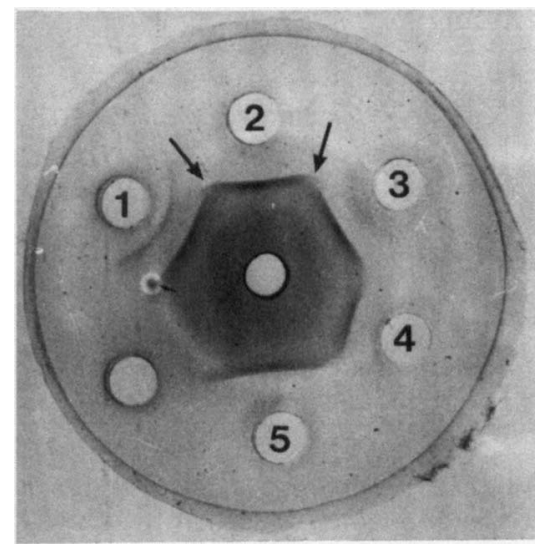

Fig. 1. Immunoprecipitation of Klebsiella K21 antiserum (centre well) with capsular polysaccharide isolated from Klebsiella 1L918 (well 1), the reference strain Klebsiella 2L222 (well 2), Klebsiella 2L229 (well 3), Klebsiella 2L230 (well 4) and Klebsiella 2L231 (well 5). Spurs, indicating partial homology between polysaccharides, are shown (arrows).

\section{Table 1. 'H NMR data for K2la polysaccharide}

'H chemical sh
(p.p.m.) at $80^{\circ}$
5.60
$5 \cdot 30$
5.09
$4.93(7)$
1.50

\begin{tabular}{cl}
$\begin{array}{c}\text { Relative } \\
\text { integrated areas }\end{array}$ & \multicolumn{1}{c}{ Assignment } \\
1 & $\mathrm{Hl \alpha}$ \\
2 & $\mathrm{H} l \alpha, \mathrm{Hl} \alpha$ \\
1 & $\mathrm{H} l \alpha$ \\
1 & $\mathrm{Hl}(\beta \mathrm{Gal}$ or $\beta \mathrm{Glc})$ \\
3 & $\mathrm{CH}_{3}$ (pyruvate)
\end{tabular}

* The value in parentheses indicates the spin coupling constant, ${ }^{3} J_{\mathrm{H}, \mathrm{H}}$, in $\mathrm{Hz}$.

\section{RESULTS}

Chromatography of $K 21$ capsular polysaccharide

Purified, hydrolysed capsular polysaccharide extracted from a number of Klebsiella strains of serotype $\mathrm{K} 21$ was analysed using paper chromatography. The polysaccharide extracted from the Klebsiella K21 reference strain 2L222 appeared to contain galactose, mannose and glucuronic acid in accordance with the reported structure for K21 antigen (Choy \& Dutton, 1973). However, polysaccharide extracted from eight other klebsiellae, including that from strain 1L918, appeared to contain galactose, glucuronic acid and rhamnose rather than mannose. We propose subsequently to refer to the material from the K21 reference Klebsiella as K21 a capsular polysaccharide, and to the novel polysaccharide that is present in $K$. pneumoniae $1 \mathrm{~L} 918$ as K21b capsular polysaccharide.

\section{Immunoprecipitation of $K 21$ capsular material}

Purified polysaccharide extracted from five Klebsiella K21 isolates was analysed using the gel diffusion technique for immunoprecipitation. Each polysaccharide was precipitated by the K21 antiserum and the gels were examined for the formation of spurs which indicate the presence of partial homology between polysaccharides. Spurs were identified between the reference Klebsiella K21a strain and all the other Klebsiella K21 isolates (Fig. 1).

\section{NMR spectroscopy of $K 21$ capsular material}

The ${ }^{1} \mathrm{H}$ NMR spectrum of $\mathrm{K} 21$ a polysaccharide at $80^{\circ} \mathrm{C}$ showed signals from five anomeric protons, integrating with respect to a three-proton methyl signal at $\sim \delta 1.5$ typical of $\mathrm{CH}_{3}$ (pyruvate). This confirmed the presence of five sugars relative to one pyruvate in the repeating unit, as observed previously (Choy \& Dutton, 1973). The anomeric proton chemical shifts (Table 1) were consistent with four $\alpha$-linked sugars and one $\beta$-linked, the spin coupling on the signal at $\delta 4.9$ being typical of $\beta$ configuration in galactose and glucose sugars. 
Table 2. 'H NMR data for the partially hydrolysed K2Ib polysaccharide

\begin{tabular}{|c|c|c|}
\hline $\begin{array}{l}{ }^{1} \mathrm{H} \text { chemical shift } \\
\text { (p.p.m.) at } 80^{\circ} \mathrm{C}^{*}\end{array}$ & $\begin{array}{c}\text { Relative } \\
\text { integrated areas }\end{array}$ & Assignment \\
\hline $\left.\begin{array}{l}5.57(3.5) \\
5.06(1.5,1.5) \\
4.93(7) \dagger \\
4.74(8) \\
1.31(6.5) \\
1.29(6.5)\end{array}\right\}$ & $\begin{array}{l}1 \\
2 \\
1 \\
1 \\
6\end{array}$ & $\begin{array}{l}\mathrm{H} 1(\alpha \mathrm{Glc} \text { or } \alpha \mathrm{Gal}) \\
\mathrm{H} 1, \mathrm{H} 12 \times(\alpha \mathrm{Rha}) \\
\mathrm{H} 1\}(\beta \mathrm{Gal} \text { or } \beta \mathrm{Glc}) \\
\mathrm{H} 1\}{ }^{2} \\
\mathrm{CH}_{3}(\alpha \mathrm{Rha}) \\
\mathrm{CH}_{3}(\alpha \mathrm{Rha})\end{array}$ \\
\hline
\end{tabular}

Table $3 .{ }^{13} C N M R$ data for the partially hydrolysed $K 21 b$ polysaccharide

\begin{tabular}{|c|c|}
\hline $\begin{array}{c}{ }^{13} \mathrm{C} \text { chemical shift } \\
\text { (p.p.m.) }\end{array}$ & Assignment \\
\hline $176 \cdot 2$ & C6 (GlcA) \\
\hline $104 \cdot 3$ & $\mathrm{Cl} 2 \times(\beta \mathrm{GlcA}$ or $\beta \mathrm{Gal})$ \\
\hline $103 \cdot 6$ & Cl $2 \times(\alpha$ Ra $)$ \\
\hline 103.0 & $\mathrm{Cl}, \mathrm{Cl}$ \\
\hline 98.9 & $\mathrm{Cl}(\alpha \mathrm{GlcA}$ or $\alpha \mathrm{Gal})$ \\
\hline 61.8 & $\mathrm{C} 6(\mathrm{Gal})$ \\
\hline $61 \cdot 5$ & $\mathrm{C} 6$ (Gal) \\
\hline $17 \cdot 5$ & $\mathrm{CH}_{3}, \mathrm{CH}_{3} 2 \times(\alpha \mathrm{Rha})$ \\
\hline
\end{tabular}

The ${ }^{1} \mathrm{H}$ NMR spectra of K21b polysaccharide isolated both by the method of Wilkinson et al. (1955) and by the method of Gotschlich et al. (1969) were distinctly different from that of K21a polysaccharide. A signal at $\delta 1.35$ was characteristic of $\mathrm{CH}_{3}$ (rhamnose) and by integration corresponded to six protons compared with $\mathrm{CH}_{3}$ (pyruvate) at $\delta 1 \cdot 5$. This suggests that there are two residues of rhamnose to one of pyruvate in the repeating structure. Rhamnose rather than fucose was the more likely assignment on the basis of the methyl chemical shift, $\mathrm{CH}_{3}$ (rhamnose) typically being observed at $\delta 1 \cdot 27-1 \cdot 36$ (Dutton \& Lim, 1985 ; Pritchard \& Furner, 1985) and $\mathrm{CH}_{3}$ (fucose) at $\delta 1 \cdot 21$ (Vliegenhart et al., 1983).

The anomeric proton signals were a broad complex pattern; this probably arose from thermal instability of the polysaccharide, since changes observed in some signals during spectral measurement at $80^{\circ} \mathrm{C}$ were irreversible with decrease in temperature. For an estimate of the number of sugars present, all the proton signals in the region $\delta 4.6-5.6$ were integrated with respect to the three methyl signals. This indicated $6 \pm 1$ sugars in the $K 21 \mathrm{~b}$ polysaccharide.

The K21b polysaccharide extracted from $K$. pneumoniae $1 \mathrm{~L} 918$ was partially hydrolysed to aid the structural determination of the repeating unit. Loss of pyruvate on hydrolysis was confirmed by the almost complete absence of a methyl signal at $\delta 1.5$ in the ${ }^{1} \mathrm{H}$ NMR (14 equivalent $\%$ remained). Two rhamnose units were still present, their methyl signals at $\delta 1.35$ each showing a spin coupling of $6.5 \mathrm{~Hz}$ typical of ${ }^{3} J_{\mathrm{CH}_{3}-\mathrm{HS}}$ in rhamnose. Unlike the native polysaccharide, a simple pattern of signals was observed in the anomeric region. Relative integrals of these signals with respect to the methyl signals of rhamnose showed that five anomeric protons were present and therefore that five sugars were in the repeating structure. Chemical shifts and spin coupling constants of the anomeric proton signals may be used to distinguish the sugars present and their anomeric configuration (Bock \& Thøgersen, 1982; Vliegenhart et al., 1983). Generally, anomeric proton chemical shifts at 5 p.p.m. and lower field indicate an $\alpha$ configuration and chemical shifts between 4.5 and 5.0 p.p.m. indicate a $\beta$ configuration. Thus, three sugars in the hydrolysed $\mathrm{K} 2 \mathrm{lb}$ polysaccharide were assigned $\alpha$ configurations and two $\beta$ configurations (Table 2). A spin coupling constant $\left({ }^{3} J_{\mathrm{H} 1-\mathrm{H} 2}\right)$ of $1.5 \mathrm{~Hz}$ is typical of rhamnose (De Bruyn et al., 1976). Therefore, from the chemical shifts of the two signals exhibiting this spin coupling, both 
(a)

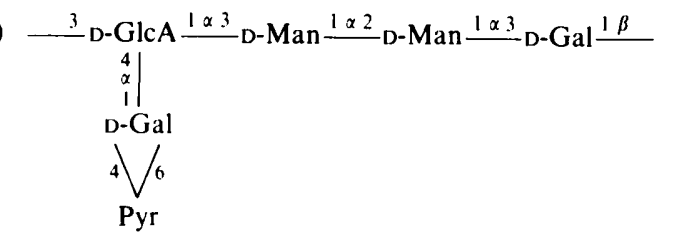

(b)

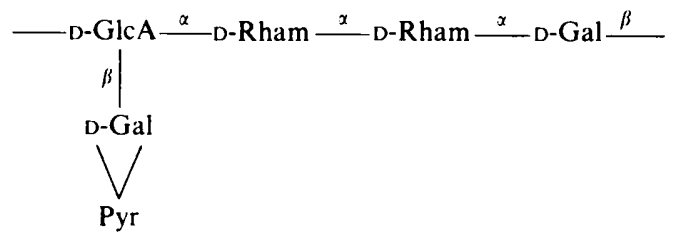

Fig. 2. (a) Oligosaccharide structure of K21a polysaccharide (Choy \& Dutton, 1973). (b) Proposed structure of the oligosaccharide repeating unit of $\mathrm{K} 21 \mathrm{~b}$ polysaccharide.

rhamnose sugars possessed $\alpha$ configuration. A spin coupling constant $\left({ }^{3} J_{\mathrm{H} 1-\mathrm{H} 2}\right)$ of $3.5 \mathrm{~Hz}$ is typical of $\alpha$ configuration in glucose and galactose and that of $8 \mathrm{~Hz}$ is typical of $\beta$ configuration in glucose and galactose. Thus the remaining three sugars present in the hydrolysed K21b polysaccharide were either glucose or galactose, one sugar being in $\alpha$ configuration and two in $\beta$ configuration. Distinction of glucose and galactose sugars in the ${ }^{1} \mathrm{H}$ NMR spectrum may be made from the magnitude of the spin coupling constant ${ }^{3} J_{\mathrm{H}_{3}-\mathrm{H} 4}$, but it was not possible to analyse fully the complex spectrum to measure this. Without full spectral analysis it was also not possible to determine whether glucuronic acid rather than glucose was present from the ${ }^{1} \mathbf{H}$ NMR alone. However, the ${ }^{1} \mathrm{H}$ NMR data supported the results obtained by paper chromatography.

${ }^{13} \mathrm{C}$ NMR of the partially hydrolysed polysaccharide (Table 3) further confirmed the assignments of the ' $\mathrm{H}$ NMR spectrum. In addition, the presence of glucuronic acid was indicated by a weak signal at 175 p.p.m. assigned to $C 6(\mathrm{GlcA})$. Also the chemical shifts of two signals at 62 p.p.m., typical of $C 6$ in hexopyranose sugars, showed that there was no substitution at $-\mathrm{OH}$ on these carbons. From paper chromatography these two sugars were galactose and from ${ }^{13} \mathrm{C}$ NMR neither sugar was linked at $\mathrm{C} 6$ in the repeating unit.

\section{DISCUSSION}

The compositions of the $\mathrm{K} 2 \mathrm{la}$ and $\mathrm{K} 2 \mathrm{lb}$ polysaccharides differ and that of $\mathrm{K} 2 \mathrm{lb}$ is consistent with the presence of two molecules of rhamnose rather than mannose in the repeating oligosaccharide unit. The high degree of cross-reactivity between the $\mathrm{K} 21 \mathrm{a}$ and the $\mathrm{K} 21 \mathrm{~b}$ polysaccharides would suggest a similar oligosaccharide structure especially with regard to the nature of the side chain. The structure of the K21 a repeated oligosaccharide (Choy \& Dutton, 1973 ) is shown in Fig. 2(a). The chromatography results for the K2l b polysaccharide material suggest the presence of glucuronic acid, galactose and rhamnose instead of mannose in the oligosaccharide structure. Thus, the structure for the repeated oligosaccharide unit of the K21 b polysaccharide may be that shown in Fig. $2(b)$. The ${ }^{1} \mathrm{H}$ NMR data were consistent with the hydrolysed $\mathrm{K} 2 \mathrm{lb}$ polysaccharide containing rhamnose, glucuronic acid and galactose in the proportions predicted in the model structure for $\mathrm{K} 21 \mathrm{~b}$, with the loss of the pyruvate moiety as a result of hydrolysis. However, because of the uncertainty in determining the number of sugars in the native $\mathrm{K} 2 \mathrm{lb}$ polysaccharide, any loss of sugar on hydrolysis is not known. In addition, the NMR data were consistent with the hydrolysed K2lb polysaccharide containing two $\beta$-linked galactose residues whereas $\mathrm{K} 21$ a polysaccharide contains only one $\beta$-linked sugar. Thus, if no sugars are lost on hydrolysis, K21b polysaccharide may possess a side chain with galactose linked to glucuronic acid by a $\beta$ rather than an $\alpha$ glycosidic bond as found in the K21a polysaccharide. 
Seven randomly selected klebsiellae provided by reference laboratories possessed the K21 btype capsular material produced by $K$. pneumoniae $1 \mathrm{~L} 918$. Only the reference Klebsiella possessed K2la-type material. Given the apparent frequency with which the K21b-type capsule arises as compared to the K21a-type capsule, it is our intention to determine whether this structural variation confers a difference in virulence, for example in resistance to phagocytosis or serum bactericidal activity. This finding also has implications for the epidemiology of infections due to Klebsiella of serotype K21 in that it is apparent that this serotype does not form a homogeneous population.

This work was supported by an MRC project grant to J.R.S. and C.A.H. We are grateful to K. Blundell, H. R. Perkins, C. Adlam and J. C. Lindon for their advice and encouragement. We thank Dr P. Mortimer, Coventry Public Health Laboratory, for the gifts of antisera and typed strains.

\section{REFERENCES}

ANONYMOUS (1974). Dyeing reagents for thin layer chromatography. Darmstadt: E. Merck.

BARR, J. G. (1981). Spontaneous loss and transfer of plasmid-mediated K21 antigen synthesis in Klebsiella pneumoniae. Journal of Clinical Pathology 34, 434-438.

Bock, K. \& THøgersen, H. (1982). Nuclear magnetic resonance spectroscopy in the study of mono- and oligosaccharides. Annual Reports on NMR Spectroscopy 13, 1-57.

Casewell, M. W. \& Talsania, H. G. (1979). Predominance of certain Klebsiella capsular types in hospitals in the United Kingdom. Journal of Infection 1, 77-79.

Сноу, Y. M. \& DutTon, G. G. S. (1973). The structure of the capsular polysaccharide from Klebsiella Ktype 21. Canadian Journal of Chemistry 51, 198-207.

De Bruyn, A., Anteunis, M., De Gussem, R. \& DutToN, G. G. S. (1976). ' $H$ N.M.R. study of Lrhamnose, methyl $\alpha$-L-rhamnopyranoside, and 4$O-\beta$-D-galactopyranosyl-L-rhamnose in deuterium oxide. Carbohydrate Research 47, 158-163.

Dutron, G. G. S. \& LiM, A. V. S. (1985). Structure of the capsular polysaccharide of Klebsiella serotype K79. Carbohydrate Research 144, 263-276.

Gotschlich, E. C., LiU, T. Y. \& ARtenstein, M. S. (1969). Human immunity to the meningococcus. III. Preparation and immunochemical properties of the group A, group B and group C meningococcal polysaccharides. Journal of Experimental Medicine 129, 1349-1356.
Luderitz, O., Staub, A. M. \& Westphal, O. (1966). Immunochemistry of $O$ and $R$ antigens of Salmonella and related Enterobacteriaceae. Bacteriological Reviews 30, 192-255.

Meers, P. D., Ayliffe, G. A. J., Emmerson, A. M., Leigh, D. A., Mayon-White, R. T., Mackingtosh, C. A. \& Strange, J. L. (1981). National Survey of Infection in Hospitals. Journal of Hospital Infections 2 (supplement), 1-39.

Nimмich, W. (1968). Zur Isolierung und qualitativen Bausteinanalyse der $\mathrm{K}$-antigene von Klebsiellen. Zeitschrift für medizinische Mikrobiologie und Immunologie 154, 117-131.

PritchaRd, D. G. \& FurNer, R. L. (1985). Structure of the group specific polysaccharide of group $E$ Streptococcus. Carbohydrate Research 144, 289-296.

SutherlaND, I. W. (1977). Bacterial exopolysaccharides - their nature and production. In Surface Carbohydrates of the Prokaryotic Cell, pp. 27-96. Edited by I. W. Sutherland. London \& New York: Academic Press.

Vliegenhart, J. F. G., Dorland, L. \& Van Halbeck, H. (1983). High resolution, ${ }^{1} \mathrm{H}$ n.m.r. spectroscopy as a tool in the structural analysis of carbohydrates related to glycoproteins. Advances in Carbohydrate Chemistry and Biochemistry 41, 221-225.

Wilkinson, J. F., Dudman, W. F. \& Aspinall, G. D. (1955). The extracellular polysaccharide of Aerobacter aerogenes A3 (S1) (Klebsiella Type 54). Biochemical Journal 59, 446-451. 